\title{
P20 Spontaneous Recanalization of the Internal Carotid Artery
}

\author{
Blanka Varnai*, Gábor Simonyi, Andrea Rapcsányi, Ervin Finta
}

Szent Imre Hospital, Budapest, Hungary

\section{ABSTRACT}

Objectives: The occlusion of the internal carotid artery (ICA) frequently leads to stroke and develops most commonly as a consequence of arterial embolism or atherosclerotic thrombosis. Following acute care, if the patient's general condition makes it possible, the patient is emitted from the hospital or, if necessary, his treatment continues in the rehabilitation department. The occlusion is generally considered irreversible. According to recent literature, spontaneous recanalization of the ICA may occur occasionally, calling for regular duplex ultrasonographic (sometimes CT angiographic) control examinations.

Methods: Authors report a case of a 67-year-old man, who suffered a right ICA occlusion and subsequent ichaemic stroke. CT angiography and Doppler ultrasound were used regularly to follow the patient's condition for 3 years later on.

Results: One year after the full occlusion of the right ICA, a spontaneous recanalization of the occlusion was observed with a $90 \%$ stenosis. In spite of the aggressive and continuous pharmacological treatment of the patient, a partial restenosis (95\%) was detected a year later, after which a successful endarterectomy was performed with permanent result.

Conclusion: The recognition and appropriate treatment of the spontaneous recanalization of the carotid artery determines the patient's long term health condition. Prospective studies are necessary to detect the frequency of occurrence of the recanalization and the relation between the therapeutic methods and the following life expectancies.

(c) 2019 Association for Research into Arterial Structure and Physiology. Publishing services by Atlantis Press International B.V. This is an open access article distributed under the CC BY-NC 4.0 license (http://creativecommons.org/licenses/by-nc/4.0/). 